\title{
Elaboración de formatos para recolección de datos de modos de fallo para el cálculo de fiabilidad en subestaciones y líneas de subtransmisión en la empresa Eléctrica de Riobamba S.A.
}

\author{
Preparation of formats for data collection of failure modes for the \\ calculation of reliability in substations and sub-transmission lines in the \\ "Empresa Eléctrica Riobamba S.A.”
}

\author{
César Marcelo Gallegos Londoño. ${ }^{1}$, Sergio Raúl Villacrés Parra. ${ }^{2}$ \& Mayra Alexandra \\ Viscaíno Cuzco. ${ }^{3}$
}

\begin{abstract}
DOI: https://doi.org/10.33262/concienciadigital.v4i1.2.1589

The aim of this research is to determine the necessary steps for data collection for the calculation of reliability indicators in urban electrical substations and urban primary feeders of the Empresa Eléctrica Riobamba S.A. Data collection is the fundamental step to obtain a correct analysis of the indicators, the results of the different analysis are directly related to the collected information credibility. The reliability of a piece of equipment depends on how it was designed, that is, on its intrinsic characteristics, a good operation and maintenance of the equipment will be reflected in a lower incidence of failure events; However, these events always happen due to deficiencies in its operation, maintenance, design, environmental conditions and normal degradation process because of the usage. To estimate reliability, it is necessary to determine with several steps: at first, an adequate equipment taxonomy and definition of the limits of each system. The second step is data collection, a good analysis depends on its quality, it is advisable to establish a good collection and a registration system always based on standardized and normalized definitions, the review of international regulations is essential to correctly establish these concepts.
\end{abstract}

\footnotetext{
1 Escuela Superior Politécnica de Chimborazo, Facultad de Mecánica. Riobamba, Ecuador. cesar.gallegos@espoch.edu.ec ORCID 0000-0002-8685-7501

2 Escuela Superior Politécnica de Chimborazo, Facultad de Mecánica. Riobamba, Ecuador. sergio.villacres@espoch.edu.ec ORCID 0000-0002-9497-9795

3 Mayra Viscaíno Cuzco. Ecuador. mayraviscaino@hotmail.com ORCID 0000-0003-4987-7797
} 
This research proposes a data collection method that can be used in methodologies such as Risk Based Inspection (RBI), Reliability Centered Maintenance (RCM), Life Cycle Costs (LCC) and RAM analysis, etc. The method will provide a very useful tool to establish strategies for the continual improvement processes and their future evaluation to verify the effectiveness of the measures taken.

Keywords: Failure modes, Reliability, RAM Analysis, data collection

\section{Resumen}

El presente estudio tuvo como propósito determinar los pasos necesarios para la recolección de datos para el cálculo de los indicadores de fiabilidad en las subestaciones eléctricas urbanas y alimentadores primarios urbanos de la Empresa Eléctrica Riobamba S.A. La recolección de datos es el paso fundamental para obtener un análisis correcto de los indicadores, los resultados de los distintos análisis están directamente relacionados con la credibilidad de la información recolectada. Un concepto fundamental es comprender que la fiabilidad de un equipo depende de cómo este fue diseñado y construido, es decir de sus características intrínsecas, una buena operación y mantenimiento de los equipos se verá reflejado en una menor incidencia de los eventos de fallo conservando su fiabilidad original, sin embargo, estos eventos siempre suceden, sea por deficiencias de mantenimiento u operación, errores en el diseño, condiciones ambientales y por el proceso normal de degradación por el uso.

Para estimar la fiabilidad es necesario cumplir con varios pasos: el primero, una adecuada taxonomía de equipos y definición de los límites de cada sistema. El segundo paso, es la recolección de datos, un buen análisis depende de la calidad de estos, es recomendable establecer un buen sistema de recolección y registro siempre basado en definiciones estandarizadas y normalizadas, la revisión de la normativa internacional es fundamental para establecer correctamente estos conceptos. Esta investigación propone un método de recolección de datos que puede ser utilizado en metodologías como el Inspección Basado en Riesco (IBR), Mantenimiento Centrado en Confiabilidad (RCM), Costos del Ciclo de Vida (LCC) y análisis RAM, etc. El método proporcionará una herramienta muy útil para establecer estrategias para la mejora continua de los procesos y su futura evaluación para constatar la eficacia de las medidas tomadas.

Palabras claves: E Modos de fallo, Fiabilidad, Análisis RAM, Recolección de datos

\section{Introducción.}

La red de transporte y distribución de energía es aquella que tiene por objeto la entrega de energía eléctrica desde las subestaciones hacia los consumidores (Alcázar Ortega, 2019). La Empresa Eléctrica Riobamba S.A. (EERSA) cuenta con 4 subestaciones urbanas (Cementerio, Salida a Guano, Parque industrial y sector Bypass) que tienen un voltaje de entrada de $69 \mathrm{kV}$ y los transforma a $13.8 \mathrm{kV}$ y $4.16 \mathrm{kV}$ para su distribución con 
potencias que varían entre 10MVA y 15MVA, cuenta con 31 alimentadores con una longitud total de $3675,28 \mathrm{~km}$

Figura 1. Esquema sistema eléctrico

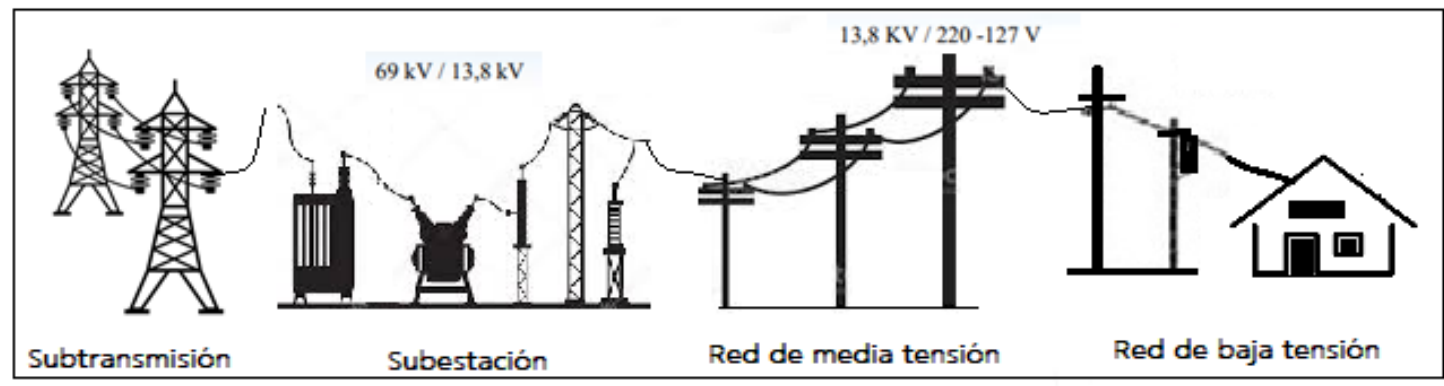

Fuente: Los autores

Elaborado por: Los autores

Los sistemas eléctricos se ven afectados por varios fenómenos que van desde elevadas temperaturas, condiciones atmosféricas, vibraciones etc. Y otras perturbaciones como transitorios, campos electromagnéticos, desbalances (Mercado \& Peña, 2016). Estos fenómenos producen condiciones como variaciones de voltaje, elevadas corrientes que provocan calentamientos que conducen a fallos que disminuye la vida útil de los equipos.

Como en cualquier tipo de activo los fallos provocan interrupciones de los sistemas de distribución eléctrico, definiéndose como "fallo" al cese de la aptitud de un elemento para cumplir su función requerida (UNE-133306 Comite Europeo de Normalización, 2018). No todos los fallos son producidos por fenómenos aleatorios, muchos de estos fallos son provocados por la inadecuada planificación y programación del mantenimiento. Desde una perspectiva práctica, el fallo se podría definir como el resultado que se origina cuando un sistema o equipo no cumple con la función que se espera que este realice en circunstancias normales de operación y considerando que se tienen listos todos los recursos necesarios para este proceso (UNE-133306 Comite Europeo de Normalización, 2018) (ESCOBAR, VILLA, \& YANEZ, 2003) (MERUANE, 2014).

Para el cálculo de la fiabilidad el parámetro fundamental es el tiempo operativo transcurrido entre dos fallos consecutivos llamado tiempo hasta el fallo (TTF) (UNE133306 Comite Europeo de Normalización, 2018) (NACHIAS, 1995). Otro concepto utilizado muy frecuentemente es el tiempo entre fallos (TBF) (MORA, Mantenimiento Industrial Efectivo, 2012), a diferencia del anterior este incluye el tiempo de reparación además del tiempo operativo entre fallos y se define como la duración del tiempo entre dos fallos consecutivos (UNE-133306 Comite Europeo de Normalización, 2018), en la Figura 2 se muestra la relación entre los dos conceptos tomados de norma ISO/TR-12489 (Modelado de confiabilidad y cálculo de sistemas de seguridad), (ISO/TR-12489, 2016). 
Figura 2. Diagrama TTF Y TBF

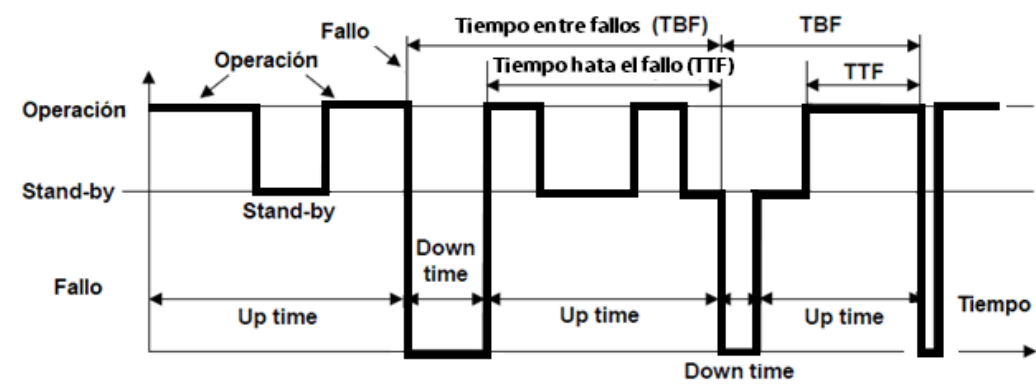

Fuente: PD CEN ISO/TR 12489:2016

Elaborado por: Los autores

La norma internacional ISO 14224 ofrece lineamientos destinados para la recolección de datos en estudios de fiabilidad y mantenimiento establecido para la industria de petróleo y gas natural (TROFFÉ, 2009), sin embargo, estos lineamientos pueden adaptarse en cualquier tipo de infraestructura, el objetivo de su uso es proporcionar estandarización, simplicidad y solidez en los casos de aplicación. Los estudios obtenidos del análisis de los datos de fiabilidad son amplios de tal suerte que se convierten en una herramienta poderosa en la toma de decisiones para mitigar el impacto que producen los fallos en disponibilidad y costos.

A diferencia de la metodología del RCM (Mantenimiento basado en la fiabilidad), la norma ISO 14224 predefine los modos de fallo por tipo de equipo lo que ahorra mucho tiempo al no listar enorme cantidad de modos de fallo asociados a las funciones de los equipos (TROFFÉ, 2009).

Según las normas internacionales la forma en que ocurre el fallo se define como "Modo de fallo" y se puede asociar al evento que hace identificable un fallo, este debe ser descrito con un sustantivo y un verbo (MOUBRAY, 2002), ejemplo (Motor quemado).

Cada modo de falla analizado tiene una causa raíz que lo origina y algunas veces más de una. La Causa raíz de un el modo de fallo se define como las circunstancias que lo provoca, estas pueden ocurrir durante las distintas fases por las que atraviesa el activo durante su ciclo de vida, como son: el diseño, la fabricación, la instalación, el montaje, la operación o mantenimiento y la desincorporación del activo. (ISO-14224, 2016).

Otro concepto fundamental es el "Mecanismo del fallo" que se define como el proceso que conduce a un fallo, este proceso puede ser físico, químico, lógico o una combinación de estos, se debe definir las causas del fallo para todo tipo de equipos (ISO-14224, 2016).

Dentro de la información asociada en cada evento del fallo, es necesario referirse al método de detección del fallo, que se define como la actividad o las actividades mediante las cuales se puede detectar que un equipo entró en un proceso de fallo (TROFFÉ, 2009) 
(HARRIS, 2000), esto es necesario para detectar al fallo en su etapa inicial, poder corregirlo y así evitar fallos catastróficos o múltiples.

El primer paso, que se realiza para un análisis de fiabilidad en equipos es elaborar un listado ordenado jerarquizado y codificado de activos (GARCIA, 2017), en la Figura 3 se muestra un ejemplo de la jerarquización.

Figura 3. Diagrama Niveles jerárquicos.

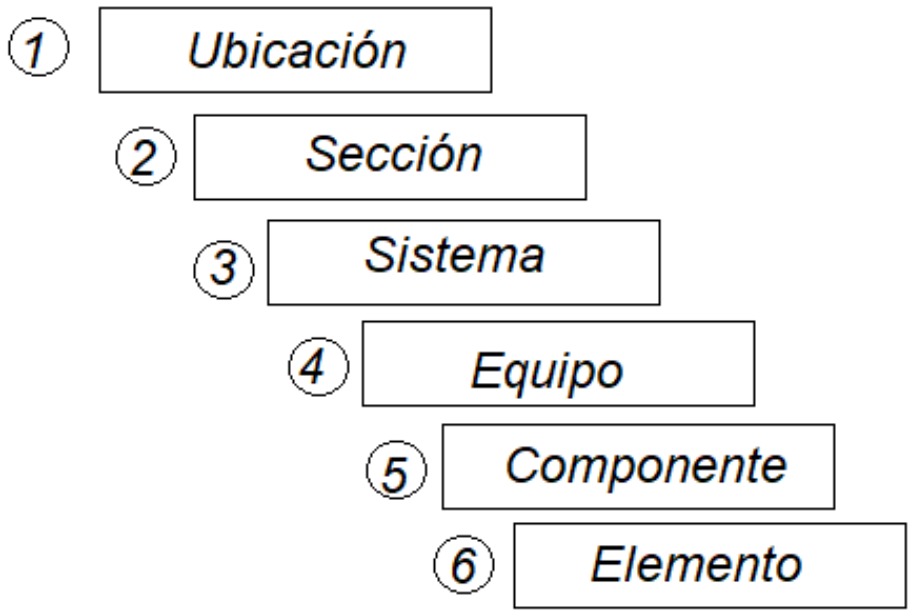

Fuente: Los Autores

Elaborado por: Los autores

En la recolección de datos para la fiabilidad se hace necesario crear familias, tipos y clases de sistemas y equipos, útil para identificar y organizar la información. Una vez organizado el inventario debe crearse un código que identifique a cada activo. Para definir las familias de equipos se tomó la clasificación en equipos: Eléctricos, Electrónicos, Mecánicos, Civiles, Instrumentación, etc. Cada familia tendrá un listado de tipos de equipos; por ejemplo, en la familia Eléctrica se van a encontrar: Disyuntores, Seccionadores, Transformadores, Pararrayos, etc. Un ejemplo se presenta en la Tabla 1.

Tabla 1. Familia y tipos de equipo

\begin{tabular}{ccccccc}
\multicolumn{2}{c}{ Familia de equipo } & \multicolumn{2}{c}{ Tipo de equipo } & & \multicolumn{2}{c}{ Clase de equipo } \\
\hline \multirow{2}{*}{ Descripción } & Código & Descripción & Código & Descripción & Código \\
\hline \multirow{2}{*}{ ELECTRICO } & E & \multirow{2}{*}{ Transformador de potencia } & TR & En aceite & 1 \\
& & & & seco & 2 \\
\hline
\end{tabular}

Fuente: Los autores

Elaborado por: Los autores

Para recoger la información útil en los determinar de los indicadores de fiabilidad, mantenibilidad y disponibilidad (RAM), corresponde definir 
tablas con los campos necesarios, la norma ISO 14224 muestra un formato para la toma de información mínima necesaria (Gallegos, Viscaíno, \& Villacrés , 2020), ver Tabla 2.

Tabla 2. Registro de datos para el cálculo de la fiabilidad, mantenibilidad y disponibilidad.

\section{Categoría de datos Datos que registrar Descripción}

\begin{tabular}{|c|c|c|}
\hline \multirow{3}{*}{ Identificar al activo } & Número del Mantenimiento & Número de identificación único \\
\hline & Código del activo & ej. Tag del equipo \\
\hline & Código del fallo (*) & $\begin{array}{l}\text { Registro del fallo (solo es relevante } \\
\text { para el mantenimiento correctivo) }\end{array}$ \\
\hline \multirow{10}{*}{$\begin{array}{l}\text { Información del } \\
\text { Mantenimiento }\end{array}$} & $\begin{array}{l}\text { Fecha de realización del } \\
\text { mantenimiento }(*)\end{array}$ & $\begin{array}{l}\text { Fecha de ejecución las tareas de } \\
\text { mantenimiento (fecha de inicio) }\end{array}$ \\
\hline & Tipo de Mantenimiento $(*)$ & $\begin{array}{l}\text { Correctiva / preventiva } \\
\text { modificativo }\end{array}$ \\
\hline & Criticidad del Mantenimiento & Alta / media / baja \\
\hline & $\begin{array}{l}\text { Tiempo planificado del } \\
\text { mantenimiento }\end{array}$ & $\begin{array}{l}\text { Relevante solo para el } \\
\text { mantenimiento preventivo }\end{array}$ \\
\hline & Tareas de Mantenimiento & $\begin{array}{l}\text { Actividad de mantenimiento (puede } \\
\text { tener instrucciones) }\end{array}$ \\
\hline & Impacto para la producción & Alta / media /baja \\
\hline & Sub-unidad atendida & $\begin{array}{l}\text { Nombre de la subunidad intervenida, } \\
\text { relevante en el mantenimiento } \\
\text { correctivo }\end{array}$ \\
\hline & Componente atendido & $\begin{array}{l}\text { Especificar el componente que } \\
\text { estuvo en mantenimiento, relevante } \\
\text { en el mantenimiento correctivo }\end{array}$ \\
\hline & Repuestos & $\begin{array}{l}\text { Disponibilidad de materiales } \\
\text { repuestos (para compra } \\
\text { existencia) }\end{array}$ \\
\hline & $\begin{array}{l}\text { Horas hombre mantenimiento, } \\
\text { por disciplina }\end{array}$ & $\begin{array}{l}\text { Horas hombre mantenimiento por } \\
\text { disciplina (mecánico, eléctrico, } \\
\text { instrumentos, otros) }\end{array}$ \\
\hline
\end{tabular}

Mantenimiento

Horas hombre totales

Total, de $\mathrm{H} / \mathrm{H}$ de mantenimiento

Fuente: Norma ISO 14224

Elaborado por: Los autores

Para el presente estudio la información de los fallos debe recolectarse en el formato de la Tabla 3. 
Tabla 3. Tabla para recolección de datos de Falla

Fuente: Los autores

\section{RECOLECCIÓN DE DATOS DE FALLO}

Mes

Semana

Subestación

Alimentador

Sistema

Equipo

Fecha y hora inicio

Fecha y hora de fin

Tiempo de paro

Descripción del fallo

Modo de fallo

Mecanismo de fallo

Subdivisión del mecanismo de

fallo

Causa de fallo

Sub causa del fallo

Impacto

Acciones correctivas

Método de detección

Elaborado por: Los autores

Para la identificación de los mecanismos de fallo mencionados anteriormente es necesario realizar una clasificación de estos, la norma ISO 14224 sugiere los siguientes:

- Fallas mecánicas

- Fallas de material

- Fallas de instrumentación

- Fallas eléctricas

- Influencia externa

- Varios

En la Tabla 4 se muestran los campos necesarios para la identificación de los mecanismos y sub-mecanismos de fallo:

Tabla 4. Mecanismos de fallo

\begin{tabular}{ccccc}
\hline \multicolumn{2}{c}{ Mecanismo de fallo } & \multicolumn{2}{c}{ Sub mecanismo de fallo } & $\begin{array}{c}\text { Descripción del } \\
\text { mecanismo de fallo }\end{array}$ \\
\cline { 1 - 3 } Código & Notación & Código & Notación & \\
\hline 1 & Fallo Eléctrico & 1.1 & Corto circuito & Cortocircuito \\
\hline
\end{tabular}

Fuente: ISO 14224

Elaborado por: Los autores 
La causa del fallo consiste en determinar el evento desencadenante que da lugar al fallo, las causas del fallo se identifican en las siguientes categorías (ISO-14224, 2016):

- Causas relacionadas con el diseño (Capacidad inapropiada / Material inapropiado)

- Causas relacionadas con la fabricación e instalación (Falla de fabricación / Falla de Instalación)

- Causas relacionadas con la operación (Error de operación / error de mantenimiento / desgaste esperado, etc.)

- Otras

Otro punto por definir son las categorías de los métodos de detección, mediante los cuales los fallos se hacen manifiestos, los métodos de detección se clasifican en las siguientes categorías (ISO-14224, 2016) (MORA, Mantenimiento Planeación, ejecución y control, 2009).

- Mantenimiento periódico

- Pruebas de correcto funcionamiento

- Inspecciones sensoriales

- Monitoreo de la condición.

Para estandarizar las acciones de mantenimiento se sugieren las siguientes categorías (ISO-14224, 2016) (HARRIS, 2000) (KNEZEVIC, 1966) (CRESPO, SÁNCHEZ, \& MOREU DE LEON, 2004):

- Lubricación

- Inspecciones sensoriales

- Mantenimiento basado en condición

- Pruebas de funcionamiento

- Reparación o reacondicionamiento

- Reemplazos

Finalmente es necesario definir las categorías de los modos de fallo a nivel 4 (equipos) para este paso se deben realizar tablas por tipo de equipo, Tabla 5.

Tabla 5. Modo de Fallo

Tipo de Equipo

Código del modo de fallo Descripción

ESO

Equipo

sobrecalentado

Fuente: Los autores

Elaborado por: Los autores 


\section{Metodología.}

La metodología para la recolección de datos para el cálculo de la fiabilidad en sistemas eléctricos se resume en los pasos que se detallan a continuación

- El primer objetivo es identificar y delimitar los sistemas de la de las subestaciones y líneas de subtransmisión para ello se debe estudiar las posibles configuraciones de las subestaciones y redes de distribución eléctrica.

- Estructurar las familias y los tipos de equipos, para ello se debe realizar listados de equipos y definir una estructura para su codificación.

- Estructurar la taxonomía de los equipos respetando los niveles jerárquicos estandarizados para cada tipo de instalación.

- Definir los parámetros para la recolección de datos para la fiabilidad en función de conceptos tomados de la normativa internacional.

- Determinar las tablas de mecanismos y sub-mecanismos de fallo.

- Determinar las tablas de causas del fallo.

- Determinar las tablas de métodos de detección del fallo.

- Determinar y clasificar los tipos de tareas de mantenimiento.

- Determinar las tablas de modos de falla para cada uno de los tipos de equipos.

\section{Resultados.}

Para realizar el inventario técnico en la Empresa Eléctrica Riobamba S.A, se delimitaron cuatro niveles jerárquicos. Ubicación es el nombre del nivel más alto, esto corresponde a todo lo que engloba la subtransmisión, en el segundo nivel jerárquico denominado Secciones les corresponden a las subestaciones eléctricas y a las líneas de subtransmisión que interconectan las subestaciones, el tercer nivel jerárquico son los sistemas en los cuales se divide cada una de las subestaciones que se mencionan a continuación:

- Barra de $69 \mathrm{k}$

- Barra de $13.8 \mathrm{KV}$

- Bahía de alimentación $69 \mathrm{kv}$

- Sistema de transformación

- Alimentadores $138 \mathrm{kv}$

- Protecciones Eléctricas

- Cuarto de control y servicios auxiliares

En el caso de las líneas de transmisión y los alimentadores son consideradas como un solo sistema, están definidos con los nombres de los lugares a los cuales llevan el servicio. Las líneas de subtransmisión que conectan las subestaciones tienen un recorrido de 138.46 km, además cuenta con 31 alimentadores con una longitud de $3675 \mathrm{~km}$. 
Los sistemas están constituidos por equipos los cuales pertenecen a la cuarta jerarquía dentro de la taxonomía, para su organización se crearon dos clasificaciones, la primera corresponde a la familia de equipos y dentro de cada familia se identifican los tipos de equipos. En las Tablas 6 y 7 se muestran las familias y tipos de equipos desarrollados para el presente estudio. Para cada familia de equipos se creó su código de identificación con un dígito alfabético.

\section{Tabla 6. Familias de equipos}

Fuente: Los autores

\begin{tabular}{lc}
\hline \multicolumn{2}{c}{ Familia de equipo } \\
\hline \multicolumn{1}{c}{ Descripción } & Código \\
\hline Eléctrico & $\mathrm{E}$ \\
Mecánico & $\mathrm{M}$ \\
Electrónico & $\mathrm{T}$ \\
Instrumentación & $\mathrm{I}$ \\
Civil & $\mathrm{C}$ \\
\hline
\end{tabular}

Elaborado por: Los autores

Dentro de cada familia se identificaron y clasificaron los tipos de equipo, para su estructura de codificación, se utilizaron dos dígitos alfabéticos que resultan ser abreviaciones de su nombre, en el caso de que en un sistema existan más de uno del mismo tipo se añaden dos dígitos numéricos secuenciales para identificarlos

Tabla 7. Tipos de equipos

\begin{tabular}{|c|c|c|c|}
\hline Descripción & Código & Descripción & Código \\
\hline Transformador & TR & Herrajes & $\mathrm{HE}$ \\
\hline Disyuntor & DI & Cable & $\mathrm{CB}$ \\
\hline $\begin{array}{l}\text { Mecanismo de } \\
\text { operación }\end{array}$ & MO & Poste & $\mathrm{PO}$ \\
\hline Seccionador & $\mathrm{SC}$ & Luminaria & LU \\
\hline Pararrayos & PA & $\begin{array}{l}\text { Seccionador } \\
\text { fusible }\end{array}$ & SF \\
\hline Barra & BA & Banco de baterías & $\mathrm{BB}$ \\
\hline Puesta a tierra & $\mathrm{TI}$ & Ups & UP \\
\hline $\begin{array}{l}\text { Transformadores de } \\
\text { corriente }\end{array}$ & $\mathrm{TC}$ & Reconectadores & $\mathrm{RC}$ \\
\hline $\begin{array}{l}\text { Transformadores de } \\
\text { voltaje }\end{array}$ & $\mathrm{TP}$ & Tablero de control & TA \\
\hline Aisladores & AI & $\begin{array}{l}\text { Banco de } \\
\text { capacitores }\end{array}$ & $\mathrm{BC}$ \\
\hline
\end{tabular}

Fuente: PD CEN ISO/TR 12489:2016

Elaborado por: Los autores

Un ejemplo de taxonomía para una subestación se muestra en la Figura 3. En el primer nivel tenemos a todo el sistema de Subtransmisión, en el segundo nivel están ubicadas las 
subestaciones eléctricas, para el tercer nivel tenemos los sistemas que componen cada subestación y finalmente en el cuarto nivel el listado de equipos que conforma cada sistema.

\section{Figura 3. Taxonomía subestación eléctrica}

\begin{tabular}{|c|c|c|c|c|c|c|c|}
\hline & & & & & & NIVEL 4 & \\
\hline & TAXONC & OMÍA & & & & S01-001-EPA01 & Pararrayos $60 \mathrm{kV}$ fase $\mathrm{A}$ \\
\hline & & & & & & S01-001-EPA02 & Pararrayos $60 \mathrm{kV}$ fase $\mathrm{B}$ \\
\hline NIVEL 1 & Subtrans & smisión & & & & S01-001-EPA03 & Pararrayos $60 \mathrm{kV}$ fase $\mathrm{C}$ \\
\hline & NIVEL 2 & Subestac & ción Cementerio & S01 & & S01-001-ESC01 & Secciona dor tripolar 69kVA \\
\hline & NIVEL 2 & Subestac & ción P. Industrial & SO2 & & S01-001-EDI01 & Disyuntor $1200 \mathrm{~A} / 72,5 \mathrm{kVA}$ \\
\hline & NIVEL 2 & Subestac & ción Bypass & $\mathrm{SO3}$ & & S01-001-EMO01 & Mecanispo de operación \\
\hline & & NIVEL 3 & Bahía & & BA01 & S01-001-ESC02 & Seccionador tripolar de barra \\
\hline & & NIVEL 3 & Sistema de transfo & nación & ST01 & & \\
\hline & & \begin{tabular}{|l|} 
NIVEL 3 \\
\end{tabular} & Alimentador & & AL01 & SO1-002-ESC01 & Secciona dor Alta tripolar 69kVA \\
\hline & & NIVEL 3 & Barra de 69 Kva & & BA01 & S01-002-EDI01 & Disyuntor 1200 A / 72,5 kVA \\
\hline & & \begin{tabular}{|l|} 
NIVEL 4 \\
\end{tabular} & Barra de $13.8 \mathrm{Kva}$ & & BA02 & \begin{tabular}{|l|} 
SO1-002-EMO01 \\
\end{tabular} & Mecanispo de operación \\
\hline & & & & & & S01-002-ETR01 & Transformador de potencia 5MVA \\
\hline & & & & & & SO1-002-ESCO2 & Secciona dor Baja tripolar 1.38kVA \\
\hline & & & & & & SO1-002-EDIO2 & Disyuntor Baja / 72,5 kVA \\
\hline & & & & & & S01-002-EMO02 & Mecanispo de operación \\
\hline & & & & & & \begin{tabular}{|l|} 
S01-002-ESCO3 \\
\end{tabular} & Seccionador tripolar de barra \\
\hline
\end{tabular}

Fuente: Los autores

Elaborado por: Los autores

Como se puede notar en el nivel cuatro de la Figura 3 se visualiza la estructura de codificación acumulada por niveles, S01 es el código de la subestación 1 para el ejemplo la Subestación Cementerio, el código a nivel de sistema es de tres dígitos de estructura numérica, 001 representa el Sistema Bahía de alimentación y 002 representa el Sistema de Transformación, a su vez el sistema 001 está formado por pararrayos, seccionadores, disyuntor y su mecanismo de operación, para el sistema 002 tenemos seccionadores, disyuntores, transformadores de potencia, etc. La estructura de codificación a nivel de equipos cuenta con cinco dígitos, el primero es la familia de equipo (E Familia eléctrica) los dos siguientes caracteres corresponden al tipo de equipo (TR Transformador de potencia), los dos últimos dígitos son numéricos, utilizados para diferenciar equipos del mismo tipo.

Un ejemplo de la recolección de datos de falla según los parámetros descritos para la fiabilidad en la Tabla 3 se muestran a continuación.

- Mes: 02

- Semana: 07

- Subestación: 02

- Sistema; Alimentador Guano

- Equipo: Reconectador RC-05

- Fecha y hora de inicio: 2020-06-18 09:45:00

_ Fecha y hora de fin: 2020-06-18 10:25:00

- Tiempo de paro: 00:40:00

- Descripción del fallo: corto circuito en la red de medio voltaje

_ Modo de fallo: DES desconocido 
- Mecanismo de fallo: fallo externo 2

- Subdivisión del mecanismo de fallo: Ambiental, viento fuerte 2.4

- Causa del fallo: general, no se conocen detalles

- Impacto: pérdida de servicio en 40 minutos

- Acciones correctivas: reinicio de reconectador

_ Método de detección: ninguna

En la Tabla 8 se muestra el desarrollo de los mecanismos de fallo para equipos eléctricos en líneas de subtransmisión y subestaciones de distribución, la tabla trae dos conceptos, el mecanismo y el sub-mecanismo de fallo, cada uno de ellos con su respectivo código, la idea de clasificar en mecanismos y submecanismos de fallo es tener más información relevante siempre y cuando haya certeza de ello, es importante que el mecanismo de fallo esté relacionado con un nivel jerárquico menor del inventario (nivel de equipo o ítem mantenible), en la práctica los mecanismos de falla representan modos de falla pero a un nivel jerárquico menor.

Tabla 8. Mecanismos de fallo

\begin{tabular}{|c|c|c|c|c|}
\hline \multicolumn{2}{|c|}{ Mecanismo de fallo } & \multicolumn{2}{|c|}{ Sub mecanismo de fallo } & \multirow{2}{*}{$\begin{array}{l}\text { Descripción del mecanismo de } \\
\text { fallo }\end{array}$} \\
\hline Código & Notación & Código & Notación & \\
\hline \multirow{14}{*}{1} & \multirow{14}{*}{$\begin{array}{l}\text { Fallo } \\
\text { Eléctrico }\end{array}$} & 1.1 & Circuito abierto & Desconexión, cable roto \\
\hline & & 1.2 & Ausencia de voltaje & Sin suministro de energía \\
\hline & & 1.3 & Falla a tierra & Baja resistencia eléctrica \\
\hline & & 1.4 & Sobrecarga & Carga mayor a la nominal \\
\hline & & 1.5 & Daño en la red & Bajantes en mal estado \\
\hline & & 1.6 & Variaciones de voltaje & $\begin{array}{l}\text { Voltajes más bajos o altos que el } \\
\text { nominal }\end{array}$ \\
\hline & & 1.7 & Cortocircuito & Cortocircuito \\
\hline & & 1.8 & Sobrecalentamiento & Conexión floja \\
\hline & & 1.9 & Equipo quemado & Pérdida del aislamiento \\
\hline & & 1.10 & $\begin{array}{l}\text { Desconexión } \\
\text { intencional }\end{array}$ & Vandalismo \\
\hline & & 1.11 & Error en la conexión & Mantenimiento incorrecto \\
\hline & & 1.12 & Capacidad insuficiente & Error en el diseño \\
\hline & & 1.13 & $\begin{array}{l}\text { Condiciones } \\
\text { atmosféricas }\end{array}$ & $\begin{array}{l}\text { Viento, descargas eléctricas, } \\
\text { lluvia }\end{array}$ \\
\hline & & 1.14 & Otro & Poste caído \\
\hline \multirow{5}{*}{2} & \multirow{5}{*}{$\begin{array}{l}\text { Influencia } \\
\text { Externa }\end{array}$} & 2.1 & Terceros & Error de operación \\
\hline & & 1.2 & Terceros & Interferencia deliberada \\
\hline & & 2.3 & Terceros & Empresas o contratistas \\
\hline & & 2.4 & Terceros & Choques de vehículos \\
\hline & & 2.4 & Ambientales & Lluvia / viento \\
\hline
\end{tabular}

Fuente: Los autores

Elaborado por: Los autores

El desarrollo de las causas de los fallos se tomó de la norma ISO 14224, el detalle se indica en la tabla 9. Las causas del fallo están relacionadas por la acción u omisión de acciones relacionadas con el personal, por eso menciona errores en el diseño, fabricación, 
operación, mantenimiento y gestión. Es muy recomendable tener causas y sub-causas del fallo para cada categoría. La descripción de las causas del fallo puede generarse en varios niveles jerárquicos dependiendo de la información disponible.

Tabla 9. Causas y sub-causas del fallo

\begin{tabular}{|c|c|c|c|c|}
\hline $\begin{array}{l}\text { Número de } \\
\text { código }\end{array}$ & Notación & $\begin{array}{l}\text { No. Código } \\
\text { de } \\
\text { subdivisión }\end{array}$ & $\begin{array}{l}\text { Subdivisión de la } \\
\text { causa de falla }\end{array}$ & Descripción de la causa de falla \\
\hline \multirow[t]{3}{*}{1} & \multirow{3}{*}{$\begin{array}{l}\text { Causas } \\
\text { concernient } \\
\text { es al diseño } \\
\text { del activo }\end{array}$} & 1 & General & $\begin{array}{l}\text { Diseño o configuración inapropiada del } \\
\text { equipo (forma, tamaño, tecnología, } \\
\text { configuración, operabilidad, } \\
\text { mantenibilidad, etc.), pero no se } \\
\text { conocen mayores detalles. }\end{array}$ \\
\hline & & 1.1 & $\begin{array}{l}\text { Capacidad } \\
\text { inapropiada }\end{array}$ & Dimensiones/capacidad inadecuada. \\
\hline & & 1.2 & $\begin{array}{l}\text { Material } \\
\text { inapropiado }\end{array}$ & Selección de materiales inapropiados. \\
\hline \multirow{3}{*}{2} & \multirow{3}{*}{$\begin{array}{l}\text { Causas } \\
\text { concernient } \\
\text { es a la } \\
\text { fabricación/ } \\
\text { instalación }\end{array}$} & 2 & General & $\begin{array}{l}\text { Falla relacionada a la fabricación o } \\
\text { instalación, pero no se conocen } \\
\text { mayores detalles. }\end{array}$ \\
\hline & & 2.1 & $\begin{array}{l}\text { Falla de } \\
\text { fabricación }\end{array}$ & Falla de fabricación o procesamiento. \\
\hline & & 2.2 & $\begin{array}{l}\text { Falla de } \\
\text { instalación }\end{array}$ & $\begin{array}{l}\text { Falla de instalación o montaje (no } \\
\text { incluye montaje después de } \\
\text { mantenimiento) }\end{array}$ \\
\hline \multirow{5}{*}{3} & \multirow{5}{*}{$\begin{array}{c}\text { Causas } \\
\text { concernient } \\
\text { es } \\
\text { al } \\
\text { mantenimie } \\
\text { nto y a la } \\
\text { operación }\end{array}$} & 3 & General & $\begin{array}{l}\text { Falla concerniente a la mala } \\
\text { operación/uso normal o } \\
\text { mantenimiento mal realizado, pero no } \\
\text { se saben muchos detalles. }\end{array}$ \\
\hline & & 3.1 & $\begin{array}{l}\text { Servicio en } \\
\text { contextos } \\
\text { operacionales } \\
\text { extremos }\end{array}$ & $\begin{array}{l}\text { Contexto operacional imprevistas, por } \\
\text { ejemplo, operación una máquina fuera } \\
\text { del rango apropiado de sus parámetros } \\
\text { normales de funcionamiento, } \\
\text { temperaturas altas. }\end{array}$ \\
\hline & & 3.2 & $\begin{array}{l}\text { Errores humanos } \\
\text { en la operación } \\
\text { de equipos }\end{array}$ & $\begin{array}{l}\text { Error humano: Error sin intención, mala } \\
\text { utilización, error por negligencia, } \\
\text { descuido en la operación, ejemplo } \\
\text { cansancio del personal }\end{array}$ \\
\hline & & 3.3 & $\begin{array}{l}\text { Errores humanos } \\
\text { en la ejecución } \\
\text { del } \\
\text { mantenimiento }\end{array}$ & $\begin{array}{l}\text { Error humano: Error sin intención, mala } \\
\text { utilización, error por negligencia, } \\
\text { descuido en la operación, ejemplo } \\
\text { cansancio del personal }\end{array}$ \\
\hline & & 3.4 & $\begin{array}{l}\text { Desgaste normal } \\
\text { por uso }\end{array}$ & $\begin{array}{l}\text { Falla por el desgaste normal en la } \\
\text { operación del equipo }\end{array}$ \\
\hline \multirow{3}{*}{4} & \multirow{3}{*}{$\begin{array}{c}\text { Falla } \\
\text { concernient } \\
\text { e a la } \\
\text { gestión del } \\
\text { mantenimie } \\
\text { nto }\end{array}$} & 4 & General & $\begin{array}{l}\text { Fallas por problemas de gestión, no se } \\
\text { saben muchos detalles. }\end{array}$ \\
\hline & & 4.1 & $\begin{array}{l}\text { Error de } \\
\text { documentación } \\
\text { mal elaborada }\end{array}$ & $\begin{array}{l}\text { Error humano: Falla en procedimientos, } \\
\text { malas especificaciones, planos no } \\
\text { actualizados, etc. Ejemplo, errores } \\
\text { debido al cansancio }\end{array}$ \\
\hline & & 4.2 & $\begin{array}{l}\text { Error por mala } \\
\text { gestión }\end{array}$ & $\begin{array}{l}\text { Falla por la mala planificación o } \\
\text { organización de las actividades, etc }\end{array}$ \\
\hline
\end{tabular}

Fuente: ISO 14224

Elaborado por: Los autores 
Los métodos de detección no es parte del desarrollo de un fallo, pero es la manera de identificar el fallo de forma temprana, de esta manera aseguramos mínimos efectos y consecuencias de los fallos. En la Tabla 10 se resumen los métodos de detección más apropiados para equipos eléctricos.

\section{Tabla 10. Métodos de detección}

\begin{tabular}{cll}
\hline Número & \multicolumn{1}{c}{ Notación } & \multicolumn{2}{c}{ Descripción } \\
\hline 1 & Mantenimiento sistemático & $\begin{array}{l}\text { Fallo manifestado durante el desarrollo del } \\
\text { mantenimiento } \\
\text { reacondicionamiento o inspección), } \\
\text { preventivo }\end{array}$ \\
$2 \quad \begin{array}{l}\text { Pruebas de correcto } \\
\text { funcionamiento }\end{array}$ & $\begin{array}{l}\text { Fallo identificado al revisar una función de un equipo } \\
\text { (detección de fallas ocultas). }\end{array}$ \\
4 & Inspecciones sensoriales & $\begin{array}{l}\text { Fallo manifiesto durante las revisiones o inspecciones } \\
\text { planificada (Inspecciones con los sentidos). } \\
\text { Fallos expuestos durante el monitoreo condicional } \\
\text { planeado programado o no, por ejemplo, termografía, } \\
\text { medición de vibraciones, análisis de aceite, etc. }\end{array}$ \\
\hline
\end{tabular}

Fuente: ISO 14224

Elaborado por: Los autores

Las actividades de mantenimiento para la corrección de fallos se estandarizan y codifican adecuadamente con el propósito de generar estadísticas que ayuden a tomar decisiones para la mejora continua. Un detalle adaptado de la norma ISO 14224 para los equipos eléctricos de subestaciones y subtransmisión se presentan en la Tabla 11.

\section{Tabla 11. Actividades de mantenimiento}

\begin{tabular}{|c|c|c|}
\hline Código & Actividad & Descripción \\
\hline 1 & Lubricación & Actividades de lubricación en general \\
\hline 2 & Inspecciones sensoriales & $\begin{array}{l}\text { Inspecciones rápidas realizadas por los } \\
\text { técnicos sin equipos complejos, basándose en } \\
\text { sus sentidos }\end{array}$ \\
\hline 3 & Mantenimiento basado en condición & $\begin{array}{l}\text { Mantenimiento periódico o en base a } \\
\text { requerimientos con investigación y medición } \\
\text { de parámetros }\end{array}$ \\
\hline 4 & Pruebas de funcionamiento & $\begin{array}{l}\text { Pruebas de funcionamiento de equipo para } \\
\text { asegurar la función }\end{array}$ \\
\hline 5 & Reparación o reacondicionamiento & Reacondicionamiento de elementos \\
\hline 6 & Reemplazos & Remplazo de componentes con uno nuevo \\
\hline
\end{tabular}

Fuente: ISO 14224

Elaborado por: Los autores

Los modos de fallo se clasifican por tipo de equipo, estos pueden generarse al no poder cumplir con la función deseada. Los modos de fallo se deben describir a nivel de equipos, un detalle de los modos de falla para equipos eléctricos se presenta en la Tabla 12. Cada modo de fallo debe estar codificado, la estructura de codificación fijada es de tres dígitos alfabéticos. Para evitar un listado grande de modos de fallo se los puede clasificar de acuerdo con los tipos de equipo que afecta. 
Tabla 12. Modos de fallo

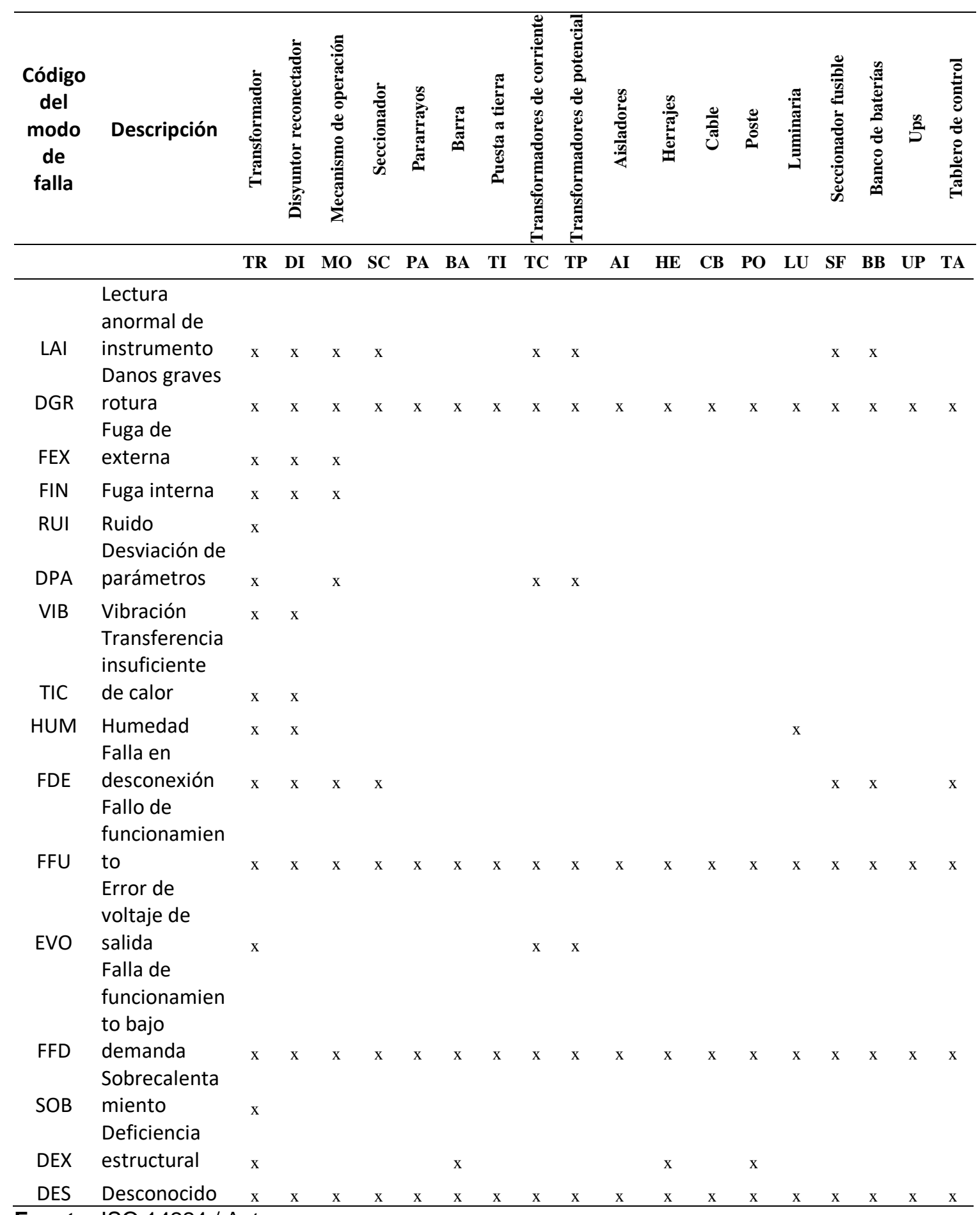

Fuente: ISO 14224 / Autores

Elaborado por: Los autores

\section{Discusión}

Las subestaciones eléctricas tienen diferentes configuraciones y montajes de acuerdo con los servicios que prestan, sin embargo, todas se pueden enfocar en los mismos criterios para la jerarquización de equipos, lo importante de este proceso es definir una estructura 
de codificación que indique de qué activo se trata y donde esté ubicado, contando siempre con un código único.

En la tabla 3 se muestra una lista de datos para recolectar información después de cada evento de fallo, este es un listado base para iniciar los cálculos de fiabilidad, permite determinar los tiempos hasta el fallo TTF, los tiempos medio hasta el fallo MTBF y los tiempos para reparar TTR, con ellos es posible calcular indicadores como la fiabilidad, mantenibilidad y disponibilidad. Sin embargo, para realizar análisis más profundos se deben aumentar más registros como los tiempos de traslado y tiempos de los retardos logísticos y administrativos que permitirán realizar nuevos análisis.

Las tablas de mecanismos de fallo son una ayuda fundamental para el registro del porqué de los fallos, la base propuesta es inicial y debe incrementarse por la presencia de nuevos casos, pero sin irse al extremo de tener demasiadas posibilidades pues se puede cometer errores en su asignación.

El criterio emitido en el párrafo anterior debe aplicarse para todas las tablas, (causa de fallos, modos de fallo, etc.)

Para analizar los métodos de identificación temprana de fallos deben participar tanto el personal de mantenimiento como el de producción, para lo cual deben tener la capacitación adecuada tanto en las técnicas como en el llenado de los registros de información.

\section{Conclusiones}

- Todas las instalaciones sufren modificaciones en el transcurso de su vida operativa, las cuales deben registrarse para tener actualizado el inventario técnico de los bienes a mantener, dentro de este proceso hay que anotar los equipos dados de baja o que han sufrido algún evento de movimiento pues estas acciones afectan a los cálculos de los indicadores de fiabilidad.

- El análisis de fallos requiere de la presentación de reportes e informes mediante la elaboración de análisis específicos para cada caso, estos análisis deben ser independientes y en relación con el contexto operacional en el que se desempeña el equipo.

- Los mecanismos de fallo analizados en cada evento deben tener un análisis forense para hallar el verdadero porqué de la falla, para ello es necesario la capacitación técnica del personal

- El propósito de este análisis no es solo el registro de datos, la principal intención es tomar las medidas necesarias para evitar que los modos de falla no vuelvan a ocurrir y minimizar sus efectos y consecuencias. Si no podemos evitar los modos de fallo la estrategia es identificarlo de manera temprana para atenderlos en su estado inicial y así evitar fallos catastróficos, lo que permitirá además ahorro de recursos. 


\section{Referencias bibliográficas.}

Alcázar Ortega, M. C. (2019). Generación, transporte y distribución de energía eléctrica. Valencia: Universidad Politécnica de Valencia.

Aldana, S., Vereda, F., Hidalgo-Alvarez, R., \& de Vicente, J. (2016). Facile synthesis of magnetic agarose microfibers by directed selfassembly. Polymer, 93, 61-64.

Bhat, S., Tripathi, A., \& Kumar, A. (2010). Supermacroprous chitosan-agarose-gelatin cryogels. in vitro characterization and in vivo assesment for cartilage tissue engineering. Journal of the Royal Society Interface, 1-15.

Bossis, G., Marins, J., Kuzhir, P., Volkova, O., \& Zubarev, A. (2015). Functionalized microfibers for field-responsive materials and biological applications. Journal of Intelligent Material Systems and Structures, 1-9.

Cortés, J., Puig, J., Morales , J., \& Mendizábal, E. (2011). Hidrogeles nanoestructurados termosensibles sintetizados mediante polimerización en microemulsión inversa. Revista Mexicana de Ingeniería Química., 10(3), 513-520.

CRESPO, A., SÁNCHEZ, A., \& MOREU DE LEON, P. (2004). Ingeniería de mantenimiento. Madrid: AENOR.

Dias, A., Hussain, A., Marcos, A., \& Roque, A. (2011). A biotechnological perspective on the application of iron oxide magnetic colloids modified with polysaccharides. Biotechnology Advances 29, 29, 142-155.

ESCOBAR, L., VILLA, E., \& YANEZ, S. (2003). Confiabilidad: Historia, estado del arte y desafíos futuros. Dyna, 70(140), 21.

Estrada Guerrero, R., Lemus Torres, D., Mendoza Anaya, D., \& Rodriguez Lugo, V. (2010). Hidrogeles poliméricos potencialmente aplicables en Agricultura. Revista Iberoamericana de Polímeros, 12(2), 76-87.

Gallegos, C., Viscaíno, M., \& Villacrés , S. (2020). Estudio de fiabilidad, mantenibilidad y disponibilidad aplicado a grupos electrógenos prime. Ambato: Ciencia Digítal.

GARCIA, O. (2017). Gestión Moderna del Mantenimiento Industrial; Principios fundamentales. Ediciones de la $\mathrm{U}$.

García-Cerda, L., Rodríguez-Fernández, O., Betancourt-Galindo, R., Saldívar-Guerrero, R., \& Torres-Torres, M. (2003). Síntesis y propiedades de ferrofluidos de magnetita. Superficies y Vacío., 16(1), 28-31.

HARRIS, M. (2000). Gestión del Mantenimiento Industrial. Madrid: Fundación REPSOL. 
Ilg, P. (2013). Stimuli-responsive hydrogels cross-linked by magnetic nanoparticles. Soft Matter, 9, 3465-3468.

ISO/TR-12489, C. (2016). Modelado de confiabilidad y cálculo de sistemas de seguridad. BSI.

ISO-14224, B. E. (2016). Recolección e intercambio de datos de confiabilidad y mantenimiento. BSI.

KNEZEVIC, J. (1966). Mantenimiento. (4ta, Ed.) Madris: Isdefe.

Lewitus, D., Branch, J., Smith, K., Callegari, G., Kohn, J., \& Neimark, A. (2011). Biohybrid carbon nanotube/agarose fibers for neural tissue engineering. Advanced Functional Materials, 21, 2624-2632.

Lin, Y.-S., Huang, K.-S., Yang, C.-H., Wang, C.-Y., Yang, Y.-S., Hsu, H.-C., . . Tsai, C.-W. (2012). Microfluidic synthesis of microfibers for magnetic-responsive controlled drug release and cell culture. PLoS ONE, 7(3), 1-8.

Mercado, V., \& Peña, J. (2016). MODELO DE GESTIÓN DE MANTENIMIENTO ENFOCADO EN LA EFICIENCIA Y OPTIMIZACIÓN DE LA ENERGÍA ELÉCTRICA. CIENCIAS BÁSICAS Y TECNOLOGÍA, 7.

MERUANE, V. (2014). Gestión de Activos. Santiago: DEMEC.

MORA, A. (2009). Mantenimiento Planeación, ejecución y control. México: Alfaomega.

MORA, A. (2012). Mantenimiento Industrial Efectivo. Envigado: Goldi.

MOUBRAY, J. (2002). Mantenimiento Centrado en la confiabilidad. Asheville: Industrias press Inc.

NACHIAS, J. (1995). Fiabilidad. Madrid: Isdefe.

Ruiz Estrada, G. (2004). Desarrollo de un Sistema de liberación de fármacos basado en nanopartículas magnéticas recubiertas con Polietilénglicol para el tratamiento de diferentes enfermedades. Madrid: Universidad Autónoma de Madrid. Departamento de Física Aplicada.

Song , J., King, S., Yoon , S., Cho, D., \& Jeong, Y. (2014). Enhanced spinnability of narbon nanotube fibers by surfactant addition. Fiberes and Polymers, 15(4), 762766.

Tartaj, P., Morales, M., González-Carreño, T., Veintemillas-Verdaguer, S., \& Serna, C. (2005). Advances in magnetic nanoparticles for biotechnology applications. Journal of Magnetism and Magnetic Materials, 290, 28-34.

TROFFÉ, M. (2009). Confiabilidad, dirigido a la gestión del conocimiento y mitigación del riesgo. Petrotecnia, 23. 
UNE-133306 Comite Europeo de Normalización. (2018). EN-UNE-133306 Terminología de mantenimiento. Madrid: AENOR.

Wulff-Pérez , M., Martín-Rodriguez, A., Gálvez-Ruiz, M., \& de Vicente, J. ( 2013 ). The effect of polymer surfactant on the rheological properties of nanoemulsions. Colloid and Polymer Science, 291, 709-716.

Zamora Mora, V., Soares, P., Echeverria, C., Hernández, R., \& Mijangos, C. (2015). Composite chitosan/Agarose ferrogels for potential applications in magnetic hyperethermia. Gels., 1, 69-80. 


\section{PARA CITAR EL ARTÍCULO INDEXADO.}

Villacrés Parra, S. R., Gallegos Londoño, C. M., \& Viscaíno Cuzco, M. A. (2021). Elaboración de formatos para recolección de datos de modos de fallo para el cálculo de fiabilidad en subestaciones y líneas de subtransmisión en la empresa Eléctrica de $\begin{array}{llll}\text { Riobamba S.A. } & \text { ConcienciaDigital, } & 4(1.2), & \text { 200-219. }\end{array}$ https://doi.org/10.33262/concienciadigital.v4i1.2.1589

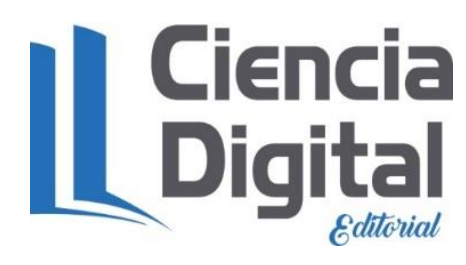

El artículo que se publica es de exclusiva responsabilidad de los autores y no necesariamente reflejan el pensamiento de la Revista Conciencia Digital.

El artículo queda en propiedad de la revista y, por tanto, su publicación parcial y/o total en otro medio tiene que ser autorizado por el director de la Revista Conciencia Digital.

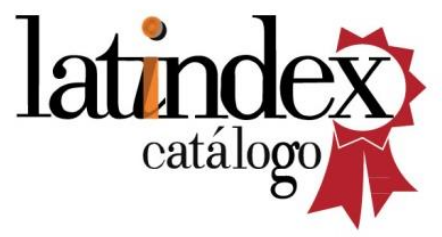

